\title{
Associations of serum fetuin-A and oxidative stress parameters with polycystic ovary syndrome
}

\author{
Sibel Sak' , Hacer Uyanikoglu', Adnan Incebiyik' ${ }^{1}$, Hatice Incebiyik², Nese Gul Hilali', Tevfik Sabuncu², Erdal Sak \\ 'Department of Obstetrics and Gynaecology, Faculty of Medicine, Harran University, Sanliurfa; ${ }^{2}$ Department of Endocrinology, Faculty of Medicine, \\ Harran University, Sanliurfa, Turkey
}

\begin{abstract}
Objective: The aim of this study was to compare serum fetuin-A levels and oxidative stress markers, as indicators of insulin resistance, in women with polycystic ovary syndrome (PCOS) and in healthy controls.

Methods: This prospective case-control study included 46 patients with PCOS and 48 age- and body mass index-matched control women. Levels of serum hormones, fetuin- $A$, and oxidative stress markers were measured in blood samples taken during the early follicular period from each participant.

Results: Follicle-stimulating hormone (FSH), luteinising hormone (LH), total testosterone levels, and the LH/FSH ratio were found to be significantly higher in women with PCOS than in controls. Serum total antioxidant status, total oxidant status, and oxidative stress index parameters all indicated significantly higher levels of oxidative stress in PCOS patients than in controls. Serum fetuin-A levels, which were analyzed as an indicator of insulin resistance, were higher in the PCOS group than in the control group $(210.26 \pm 65.06 \mu \mathrm{g} / \mathrm{mL}$ and $182.68 \pm 51.20 \mu \mathrm{g} / \mathrm{mL}$, respectively; $p=0.024$ ).

Conclusion: The data obtained from the present study suggest that higher levels of both serum fetuin-A and oxidative stress markers might be related with PCOS.
\end{abstract}

Keywords: Fetuin-A; Oxidative stress; Polycystic ovary syndrome

\section{Introduction}

Polycystic ovary syndrome (PCOS) is the most frequently seen endocrinopathy in females of reproductive age. Although figures vary according to the diagnostic criteria, its incidence is reported to be $6 \%$ to $20 \%$ [1-3]. Acne, hirsutism, seborrhoea, menstrual irregularities, anovulation, infertility, obesity, and several metabolic (e.g., glucose and lipid abnormalities) and atherosclerotic disorders are among the symptoms and signs of this disease [4,5]. With regard to the aetiology of PCOS, some causative factors such as hormonal disorders, adipose

Received: Jan 12, 2018· Revised: Jun 19, 2018 · Accepted: Jun 22, 2018 Corresponding author: Hacer Uyanikoglu

Department of Obstetrics and Gynaecology, Faculty of Medicine, Harran University, Sanliurfa 63200, Turkey

Tel:+90-0535-5740425 Fax:+90-414-318-3192 E-mail:huoglu@hotmail.com

This is an Open Access article distributed under the terms of the Creative Commons Attribution Non-Commercial License (http://creativecommons.org/licenses/by-nc/4.0/) which permits unrestricted non-commercial use, distribution, and reproduction in any medium, provided the original work is properly cited. tissue dysfunction, and oxidative stress have been blamed, but a definitive consensus has not been reached [6]. However, in recent years, insulin resistance (IR) has been reported to play an important role in the progression of $\mathrm{PCOS}[1,7,8]$.

Fetuin-A is a glycoprotein produced in the liver and released into the plasma [9]. It is known to have various biological functions, including the prevention of tissue calcification and maintenance of calcium-phosphorus balance $[10,11]$. Data obtained in recent experimental studies showed that elevated levels of fetuin-A lead to IR by disturbing insulin receptor signalling [11].

Oxidative stress has been defined as an imbalance between the formation of reactive oxygen species, which cause oxidative damage, and the antioxidant defence system $[12,13]$. The molecular damage caused by free radicals has been asserted to lead to many diseases, such as atherosclerosis, diabetes mellitus, and PCOS [12].

Considering all of this, IR and oxidative stress may play important roles in the development of PCOS. A literature review spanning the 
last few decades revealed scarce documentation of the role of fetuinA and oxidative stress parameters in PCOS. Thus, the present study sought to evaluate fetuin- $A$ and oxidative stress parameters in patients with PCOS.

\section{Methods}

The present case-control study was carried out in the Obstetrics and Gynaecology Department of the Harran University Medical Faculty from April 2013 to June 2015. The study was planned in accordance with the second Helsinki Declaration (2008) and approved by the university's Ethics Committee following a review of the study protocol. All participants were informed about the study and gave their written consent before participating in the study.

It was determined (using the $\mathrm{G}^{*}$ Power 3 power-analysis program; Heinrich-Heine-Universität Düsseldorf, Düsseldorf, Germany) that at least 72 subjects (at least 36 subjects in each group) were required for a two-tailed a-value of 0.05 (sensitivity, $95 \%$ ) and a $\beta$-value of 0.20 (study power, 80\%; effect size, 0.60) [14].

Our study included a total of 94 women, including 46 with PCOS and 48 healthy controls. After a detailed medical history was obtained from all participants, physical examinations and ultrasonographic evaluations were carried out, blood samples were obtained for biochemical testing. Body mass index (BMI) was calculated as weight $(\mathrm{kg})$ divided by height squared $\left(\mathrm{m}^{2}\right)$.

PCOS was diagnosed based on the presence of at least two of the following three clinical and laboratory criteria adopted from the 2003 Rotterdam criteria: (1) oligo- or anovulation, (2) positive clinical or biochemical indicators of hyperandrogenism, and (3) a polycystic ovarian appearance on an ultrasonographic examination [7]. Because of the small number of patients, we did not divide them by PCOS phenotype.

\section{Inclusion criteria}

The PCOS patients included those diagnosed using the Rotterdam criteria. The control group included 48 women who received routine gynaecological examinations and agreed to participate in the study. The control group subjects were matched for age, sex, and BMI with the PCOS patients.

\section{Exclusion criteria}

Patients with congenital adrenal hyperplasia, hyperprolactinaemia, an androgen-secreting tumour, or thyroid dysfunction, as well as those who used oral contraceptives, were excluded from the study. In addition, because of the possibility of effects on oxidative stress parameters, we excluded patients with chronic disease (including hypertension, diabetes mellitus, and pulmonary, liver, or renal dis- eases), who smoked, or who used antioxidant supplements (vitamins $\mathrm{C}$, E, etc.). We did not perform an oral glucose tolerance test, but we excluded patients with fasting glucose levels higher than $100 \mathrm{mg} / \mathrm{dL}$.

\section{Protocol for obtaining serum samples}

Blood samples were obtained through plastic injectors from the antecubital vein during the patient's early follicular phase (spontaneously or on the 2nd or 3rd day of menstruation induced by medication) in the morning after overnight fasting. Each blood sample was $10 \mathrm{~mL}$ in total; the first $5 \mathrm{~mL}$ was allocated for the laboratory testing and the remaining $5 \mathrm{~mL}$ was placed in polypropylene tubes. Within 30 minutes after being drawn, the blood samples were centrifuged at 3,000 rpm for 10 minutes. The supernatant serum samples were placed in plastic tubes and stored at $-80^{\circ} \mathrm{C}$ until the evaluation. Because of the characteristics of the study, no preservative substance was added to the samples. Fasting glucose, luteinising hormone ( $\mathrm{LH})$, follicle-stimulating hormone (FSH), total testosterone, dehydroepiandrosterone sulphate (DHEA-S), and oestradiol levels were measured with routine laboratory techniques.

\section{Analysis of serum oxidative stress parameters}

Measurements of serum oxidative stress parameters, including total antioxidant status (TAS), total oxidant status (TOS), and the oxidative stress index (OSI), were carried out as described in our previous studies $[15,16]$.

\section{1) Measurement of TAS}

TAS was measured with the spectrophotometric method using the Rel Assay commercial kit (Rel Assay Diagnostics, Gaziantep, Turkey). The results are reported as millimoles of Trolox equivalents per litre (mmol Trolox equivalent/L).

\section{2) Measurement of TOS}

TOS were measured with the spectrophotometric method using the Rel Assay Diagnostics commercial kit (Rel Assay Diagnostics). The results are presented as micromoles of hydrogen peroxide per litre ( $\mu \mathrm{mol} \mathrm{H}_{2} \mathrm{O}_{2}$ equivalent/L).

\section{3) OSI calculation}

OSI was defined as the percentage ratio of TOS to TAS.

\section{4) Measurement of fetuin- $A$}

Serum fetuin-A levels were measured with enzyme-linked immunosorbent assay kits, according to the manufacturer's instructions (BioVendor Laboratory Medicine, Brno, Czech Republic). The antibodies were specific for the human fetuin-A protein, with an assay sensitivity of $3.5 \mu \mathrm{g} / \mathrm{mL}$. 


\section{Statistical analysis}

The statistical analysis was performed using SPSS ver. 16.0 (SPSS Inc., Chicago, IL, USA). All data are presented as mean \pm standard deviation. The Kolmogorov-Smirnov test was applied to analyze the distribution of data. The independent-samples $t$-test was used to analyze between-group differences in quantitative parameters with a normal distribution of data, and the Mann-Whitney U-test was used for data without a normal distribution. The Pearson correlation test was applied to analyze correlations between the data. Multiple linear regression analysis was used to determine the effects of the independent variables on the dependent variables. The $p$-values of $<0.05$ were considered to indicate statistical significance.

\section{Results}

The demographic and basic laboratory data of the groups are presented in Table 1. No significant differences were identified between the groups in terms of age, BMl, and levels of glucose and DHEA-S. However, FSH, LH, total testosterone levels, and the LH/FSH ratio were found to be significantly higher in patients with PCOS than in the

Table 1. Clinical characteristics of the PCOS and control groups

\begin{tabular}{lccr} 
Characteristics & $\begin{array}{c}\text { PCOS group } \\
(\mathrm{n}=46)\end{array}$ & $\begin{array}{c}\text { Control group } \\
(\mathrm{n}=48)\end{array}$ & $p$-value \\
\hline Age $(\mathrm{yr})$ & $23.45 \pm 3.76$ & $23.77 \pm 3.82$ & 0.688 \\
BMI $\left(\mathrm{kg} / \mathrm{m}^{2}\right)$ & $29.50 \pm 3.65$ & $28.61 \pm 3.86$ & 0.257 \\
Glucose $(\mathrm{mg} / \mathrm{dL})$ & $86.97 \pm 11.15$ & $83.44 \pm 7.39$ & 0.072 \\
FSH $(\mathrm{mlU} / \mathrm{mL})$ & $5.88 \pm 0.58$ & $5.60 \pm 0.38$ & 0.006 \\
LH $(\mathrm{mlU} / \mathrm{mL})$ & $9.04 \pm 1.27$ & $7.47 \pm 1.40$ & $<0.001$ \\
LH/FSH ratio & $1.55 \pm 0.26$ & $1.34 \pm 0.27$ & $<0.001$ \\
Oestradiol $(\mathrm{pg} / \mathrm{mL})$ & $59.53 \pm 12.38$ & $55.36 \pm 11.53$ & 0.095 \\
Total testosterone $(\mathrm{ng} / \mathrm{mL})$ & $0.64 \pm 0.23$ & $0.45 \pm 0.06$ & $<0.001$ \\
DHEA-S $(\mathrm{ng} / \mathrm{mL})$ & $1,765.15 \pm 434.72$ & $1,720.20 \pm 371.68$ & 0.591
\end{tabular}

Values are presented as mean \pm standard deviation.

PCOS, polycystic ovary syndrome; BMI, body mass index; FSH, follicle-stimulating hormone; LH, luteinising hormone; DHEA-S, dehydroepiandrosterone sulphate.

Table 2. Comparison of oxidative stress and fetuin-A levels in both groups

\begin{tabular}{lccc}
\hline Variable & $\begin{array}{c}\text { PCOS group } \\
(n=46)\end{array}$ & $\begin{array}{c}\text { Control group } \\
(n=48)\end{array}$ & $p$-value \\
\hline TAS (mmol Trolox equivalent/L) & $0.87 \pm 0.12$ & $0.95 \pm 0.17$ & 0.009 \\
TOS ( $\mu$ mol $\mathrm{H}_{2} \mathrm{O}_{2}$ equivalent/L) & $30.38 \pm 7.72$ & $26.56 \pm 6.41$ & 0.002 \\
OSI & $3.47 \pm 1.00$ & $2.88 \pm 0.96$ & 0.004 \\
Fetuin-A $(\mu \mathrm{g} / \mathrm{mL})$ & $210.26 \pm 65.06$ & $182.68 \pm 51.20$ & 0.024
\end{tabular}

Values are presented as mean \pm standard deviation.

PCOS, polycystic ovary syndrome; TAS, total antioxidant status; TOS, total oxidant status; OSI, oxidative stress index. controls ( $p=0.006$ for FSH and $p<0.001$ for others) (Table 1).

TOS and the OSI were also determined to be significantly higher in the PCOS group than in the control group $(p=0.002, p=0.004$, respectively) (Table 2). Moreover, TAS levels were significantly lower in patients with PCOS than in the controls ( $p=0.009$ ) (Table 2 ), which is consistent with the PCOS group experiencing higher levels of overall oxidative stress.

Serum fetuin-A levels were higher in the PCOS group than in the control group $(210.26 \pm 65.06 \mu \mathrm{g} / \mathrm{mL}$ and $182.68 \pm 51.20 \mu \mathrm{g} / \mathrm{mL}$, respectively; $p=0.024)$. A receiver operating characteristic $(\mathrm{ROC})$ analysis was performed, as shown in Figure 1. The area under the curve was 0.68 (95\% confidence interval, $0.58-0.79 ; p=0.002$ ). According to the ROC analysis, the cut-off value of $179.5 \mu \mathrm{g} / \mathrm{mL}$ for fetuin-A had a sensitivity of $72 \%$ and specificity of $59 \%$. When we used a cutoff

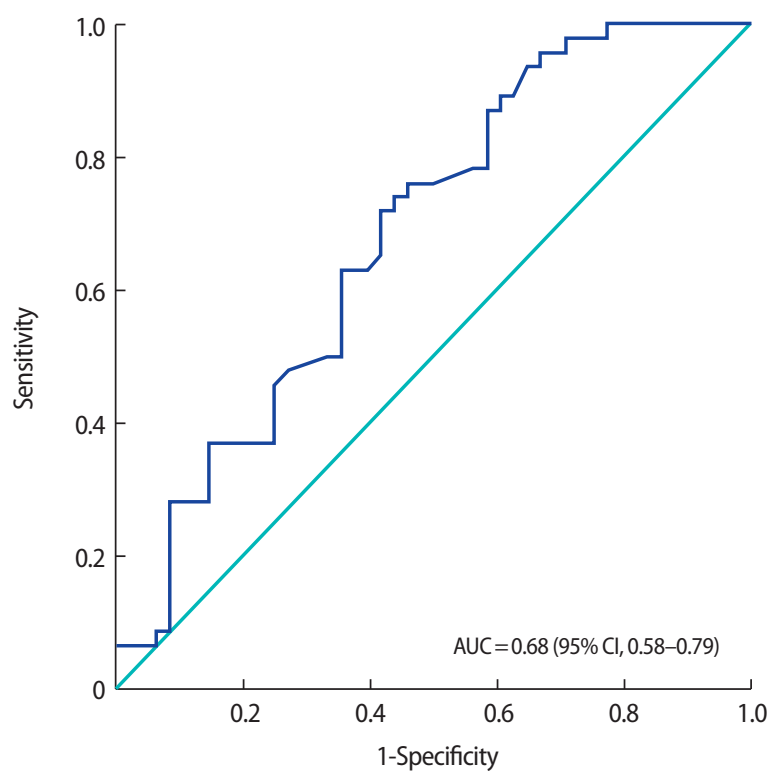

Figure 1. Receiver operating characteristic curve analysis for serum fetuin-A levels in polycystic ovary syndrome patients (respective cutoff, area under the curve [AUC], and sensitivity and specificity values). $\mathrm{Cl}$, confidence interval.

Table 3. Correlations of serum fetuin-A levels with $\mathrm{LH}$ levels, the $\mathrm{LH} /$ FSH ratio, glucose levels, TAS, TOS, and BMI

\begin{tabular}{lcc}
\hline \multirow{2}{*}{ Variable } & \multicolumn{2}{c}{ Serum fetuin-A level } \\
\cline { 2 - 3 } & $r$ & $p$-value \\
\hline LH & 0.277 & 0.007 \\
LH/FSH ratio & 0.257 & 0.012 \\
Glucose level & 0.229 & 0.027 \\
TAS & -0.134 & 0.197 \\
TOS & 0.024 & 0.818 \\
BMI & 0.181 & 0.082 \\
\hline
\end{tabular}

$\mathrm{LH}$, luteinising hormone; $\mathrm{FSH}$, follicle-stimulating hormone; TAS, total antioxidant status; TOS, total oxidant status; BMI, body mass index. 
Table 4. Correlations of serum oxidative stress markers with age, BMl, glucose levels, LH, FSH, and the LH/FSH ratio

\begin{tabular}{|c|c|c|c|c|c|c|}
\hline \multirow{2}{*}{ Variable } & \multicolumn{2}{|c|}{ TAS } & \multicolumn{2}{|c|}{ TOS } & \multicolumn{2}{|c|}{ OSI } \\
\hline & $r$ & $p$-value & $r$ & $p$-value & $r$ & $p$-value \\
\hline Age & 0.071 & 0.11 & -0.165 & 0.11 & -0.162 & 0.110 \\
\hline BMI & 0.032 & 0.76 & -0.025 & 0.81 & -0.046 & 0.650 \\
\hline Glucose level & -0.066 & 0.52 & 0.258 & 0.01 & 0.196 & 0.050 \\
\hline LH & -0.138 & 0.18 & 0.257 & 0.01 & 0.229 & 0.003 \\
\hline FSH & 0.157 & 0.13 & -0.011 & 0.91 & -0.118 & 0.257 \\
\hline LH/FSH ratio & -0.280 & 0.01 & 0.264 & 0.01 & 0.341 & 0.001 \\
\hline
\end{tabular}

BMI, body mass index; LH, luteinising hormone; FSH, follicle-stimulating hormone; TAS, total antioxidant status; TOS, total oxidant status; OSI, oxidative stress index.

value of $189 \mu \mathrm{g} / \mathrm{mL}$ for fetuin-A, the values of sensitivity and specificity were $63 \%$ and $65 \%$, respectively.

Significant positive correlations were identified between levels of fetuin- $A$ and levels of $L H$, the LH/FSH ratio, and glucose levels. However, there was no correlation between fetuin-A levels and oxidative stress parameters (i.e., TAS, TOS, and OSI) (Table 3). Additionally, we performed a correlation analysis between the oxidative stress parameters (TAS, TOS, and OSI) and age, BMI, glucose, LH, FSH, and the LH/FSH ratio. There were significant positive correlations between TOS and glucose, $\mathrm{LH}$, and the $\mathrm{LH} / \mathrm{FSH}$ ratio $(r=0.258, r=0.257$, and $r=0.264$, respectively; all $p=0.01$ ). Moreover, a significant positive correlation was found between the OSI and LH levels and the LH/FSH ratio $(r=0.229$, $p=0.003$, and $r=0.341, p=0.001$, respectively). TAS showed a negative correlation with the LH/FSH ratio $(r=-0.28, p=0.01)$ (Table 4).

In order to exclude several possible confounding factors (i.e., age, BMl, glucose, LH, FSH, and the LH/FSH ratio), we applied multiple regression analysis. According to the regression analysis, TOS levels were only significantly correlated with glucose levels $(t=2.03, p=0.04)$. The OSI was only significantly associated with the LH/FSH ratio $(t=2.59, p=0.01)$.

\section{Discussion}

The data obtained from the present study, which evaluated levels of fetuin-A and oxidative stress markers in patients with PCOS, showed that serum levels of fetuin-A were significantly higher in patients with PCOS than in controls. We evaluated the correlations between fetuin-A and BMl, oxidative stress parameters, the LH/FSH ratio, serum levels of $\mathrm{LH}$ and FSH, and glucose levels in all participants. As a result, we found significant positive correlations between levels of serum fetuin-A and levels of $\mathrm{LH}$, the LH/FSH ratio, and glucose levels, but there were no correlations between fetuin-A levels and levels of serum FSH or oxidative stress markers (TAS and TOS).

The literature contains limited data about the role of fetuin-A in patients with PCOS. Recently, some researchers have reported that fetuin-A may be a useful biochemical indicator for detecting IR in PCOS patients [11,17-19]. A high level of fetuin-A in the body has been as- serted to be connected with IR, via inhibition of the autophosphorylation and tyrosine kinase activity of insulin receptors in the liver and muscle tissues $[18,19]$. Eventually, ongoing IR leads to hyperinsulinaemia. Increased levels of insulin have been shown to cause both a surge in $\mathrm{LH}$ and increased androgen release from the theca cells through the ovarian insulin receptors in women with PCOS. Finally, the clinical picture involves the appearance of hyperandrogenaemia, one of the symptoms of PCOS $[2,19,20]$. However, the relationship between fetuin- $A$ and IR remains controversial. In contrast to statements in some articles describing a significant relationship between fetuin-A and IR [19,21,22], Gulhan et al. [20] reported that there was no such relationship. They suggested that rather than IR, genetic factors and several cytokines released from adipose tissue could have an effect on the release of fetuin- $A$ [20].

Approximately $50 \%$ of women with PCOS are overweight or obese, and most of them have severe IR and excess androgen [23]. In our study, there was no correlation between BMI and serum fetuin-A levels in either group. Similarly, Kozakowski et al. [18] showed that serum fetuin-A levels in both lean and obese PCOS patients were not significantly different. In contrast to our findings, they found an association between fetuin-A levels and DHEA-S, but not the LH/FSH ratio. We found higher fetuin-A levels in PCOS patients. Additionally, positive correlations were found between levels of serum fetuin- $A$ and levels of $\mathrm{LH}$, the $\mathrm{LH} / \mathrm{FSH}$ ratio, and serum glucose levels, but no correlation with DHEA-S was found in this study. The dissimilarity between our findings and those of Kozakowski et al. [18] may have been due to the use of different study groups, in that they had only PCOS patients, while we had both PCOS and healthy control subjects, and we included more participants.

One of the basic characteristics of PCOS is increased serum levels of oxidative stress markers [12]. We demonstrated in a previous study that increased oxidative stress in the body could contribute to the pathogenesis of PCOS [24]. Although no consensus has been reached the formation of oxidative stress, one theory is related to the increased number of mononuclear cells that develop secondarily to hyperglycaemia in PCOS patients. Increased amounts of mononucle- 
ar cells cause enhanced production of inflammatory cytokines and free oxygen radicals [25]. Molecular damage due to free oxygen radicals has been suggested to play an important role in the aetiopathogenesis of PCOS [26]. In this study, TOS and the OSI in the PCOS group were significantly higher than in the control group, which is in agreement with the literature. Moreover, levels of TAS, which are an indicator of levels of antioxidants that participate actively in the elimination of free oxygen radicals in the body, were significantly lower in the PCOS patients than in the controls.

There is no universally agreed-upon treatment for PCOS because its aetiology is not clearly understood. Hence, there is a continued need for studies on the aetiopathogenesis of this disease. The present study suggests that higher levels of serum fetuin-A and oxidative stress markers might be associated with PCOS.

\section{Conflict of interest}

No potential conflict of interest relevant to this article was reported.

\section{Acknowledgments}

The authors would like to thank Mehmet Cebel (Cebel Chemistry) and Mehmet Demir (Yildiz Chemistry), Sanliurfa, Turkey.

\section{References}

1. Williams T, Mortada R, Porter S. Diagnosis and treatment of polycystic ovary syndrome. Am Fam Physician 2016;94:106-13.

2. Tokmak A, Kokanali D, Timur H, Kuntay Kokanali M, Yilmaz N. Association between anti-Mullerian hormone and insulin resistance in non-obese adolescent females with polycystic ovary syndrome. Gynecol Endocrinol 2016;32:926-30.

3. Graff SK, Mario FM, Ziegelmann P, Spritzer PM. Effects of orlistat vs. metformin on weight loss-related clinical variables in women with PCOS: systematic review and meta-analysis. Int J Clin Pract 2016;70:450-61.

4. Layegh P, Mousavi Z, Farrokh Tehrani D, Parizadeh SM, Khajedaluee $M$. Insulin resistance and endocrine-metabolic abnormalities in polycystic ovarian syndrome: comparison between obese and non-obese PCOS patients. Int J Reprod Biomed (Yazd) 2016; 14:263-70.

5. Nasrat H, Patra SK, Goswami B, Jain A, Raghunandan C. Study of association of leptin and insulin resistance markers in patients of PCOS. Indian J Clin Biochem 2016;31:104-7.

6. Papalou O, Victor VM, Diamanti-Kandarakis E. Oxidative stress in polycystic ovary syndrome. Curr Pharm Des 2016;22:2709-22.

7. Rotterdam ESHRE/ASRM-Sponsored PCOS Consensus Workshop
Group. Revised 2003 consensus on diagnostic criteria and longterm health risks related to polycystic ovary syndrome. Fertil Steril 2004;81:19-25.

8. Barber TM, Dimitriadis GK, Andreou A, Franks S. Polycystic ovary syndrome: insight into pathogenesis and a common association with insulin resistance. Clin Med (Lond) 2016;16:262-6.

9. Voelkl J, Pakladok T, Lin Y, Viereck R, Lebedeva A, Kukuk D, et al. Up-regulation of hepatic alpha-2-HS-glycoprotein transcription by testosterone via androgen receptor activation. Cell Physiol Biochem 2014;33:1911-20.

10. Robinson KN, Teran-Garcia M. From infancy to aging: biological and behavioral modifiers of fetuin-A. Biochimie 2016;124:141-9.

11. Abali R, Celik C, Tasdemir N, Guzel S, Alpsoy S, Yuksel A, et al. The serum protein a2-Heremans-Schmid glycoprotein/fetuin-A concentration and carotid intima-media thickness in women with polycystic ovary syndrome. Eur J Obstet Gynecol Reprod Biol 2013;169:45-9.

12. Kucukaydın Z, Duran C, Basaran M, Camlica F, Erdem SS, Basaran $A$, et al. Plasma total oxidant and antioxidant status after oral glucose tolerance and mixed meal tests in patients with polycystic ovary syndrome. J Endocrinol Invest 2016;39:1139-48.

13. Demir B, Cengiz H, Ungan I, Gedikbasi A, Karakoc G, Demir E, et al. The relationship between epicardial adipose tissue thickness and oxidative stress parameters in patients with isolated polycystic ovary syndrome. Gynecol Endocrinol 2015;31:531-5.

14. Faul F, Erdfelder E, Lang AG, Buchner A. G*Power 3: a flexible statistical power analysis program for the social, behavioral, and biomedical sciences. Behav Res Methods 2007;39:175-91.

15. Incebiyik A, Camuzcuoglu H, Vural M, Hilali NG, Camuzcuoglu A, Seker $A$, et al. Assessment of apoptotic activity dysregulation and oxidative stress in the development of epithelial ovarian cancer: a case-controlled descriptive analysis. Gynecol Obstet Invest 2016;81:71-7.

16. Incebiyik A, Vural M, Camuzcuoglu H, Taskin A, Camuzcuoglu A, Hilali NG, et al. Can circulating M30 and M65 levels be beneficial markers in the diagnosis and management of patients with complete hydatidiform mole? Wien Klin Wochenschr 2016;128(Suppl 8):566-71.

17. Hizli S, Abaci A, Ozdemir O, Akelma Z, Akin O. Relation of fetuin A levels with cardiac, subcutaneous lipid accumulation and insulin resistance parameters in Turkish obese children. J Pediatr Endocrinol Metab 2016;29:669-73.

18. Kozakowski J, Jeske W, Zgliczynski W. Fetuin-A levels in lean and obese women with polycystic ovary syndrome. Endokrynol Pol 2014;65:371-6.

19. Enli Y, Fenkci SM, Fenkci V, Oztekin O. Serum fetuin-A levels, insulin resistance and oxidative stress in women with polycystic ova- 
ry syndrome. Gynecol Endocrinol 2013;29:1036-9.

20. Gulhan I, Bozkaya G, Oztekin D, Uyar I, Kebapcilar AG, Pamuk B. Serum fetuin-A levels in women with polycystic ovary syndrome. Arch Gynecol Obstet 2012;286:1473-6.

21. Ou HY, Yang YC, Wu HT, Wu JS, Lu FH, Chang CJ. Serum fetuin-A concentrations are elevated in subjects with impaired glucose tolerance and newly diagnosed type 2 diabetes. Clin Endocrinol (Oxf) 2011;75:450-5.

22. Yin L, Cai WJ, Chang XY, Li J, Su XH, Zhu LY, et al. Association between fetuin-A levels with insulin resistance and carotid intimamedia thickness in patients with new-onset type 2 diabetes mellitus. Biomed Rep 2014;2:839-42.

23. Lim SS, Norman RJ, Davies MJ, Moran LJ. The effect of obesity on polycystic ovary syndrome: a systematic review and meta-analysis. Obes Rev 2013;14:95-109.

24. Uyanikoglu H, Sabuncu T, Dursun H, Sezen H, Aksoy N. Circulating levels of apoptotic markers and oxidative stress parameters in women with polycystic ovary syndrome: a case-controlled descriptive study. Biomarkers 2017;22:643-7.

25. Isik H, Aynioglu O, Timur H, Sahbaz A, Harma M, Can M, et al. Is xanthine oxidase activity in polycystic ovary syndrome associated with inflammatory and cardiovascular risk factors? J Reprod Immunol 2016;116:98-103.

26. Zuo T, Zhu M, Xu W. Roles of oxidative stress in polycystic ovary syndrome and cancers. Oxid Med Cell Longev 2016;2016:8589318. 